\title{
Symbol timing for multiple antenna OFDM systems
}

\author{
Tim C.W. Schenk, Maurice M. de Laat, Peter F.M. Smulders and Erik R. Fledderus \\ Eindhoven University of Technology, Radiocommunication Chair, PO Box 513, 5600 MB Eindhoven, The Netherlands, \\ Tel. +31 40247 3436, Fax. +31 40245 5197, timschenk@ieee.org.
}

\begin{abstract}
Accurate symbol timing in multiple-input multipleoutput (MIMO) OFDM systems allows for guard-intervals (GIs), which are short compared to the channel's rms delay spread. These short GIs improve the effective throughput of these kind of systems. This paper proposes different timing approaches for MIMO OFDM systems, which apply knowledge about the channel impulse response. The techniques differ in complexity and performance. From a numerical performance study it is concluded that for single-cluster channels and moderate delay spreads the low-complex algorithms can be applied without considerable loss in performance. For high delay spreads and multiple clusters, however, the proposed algorithm, which attempts to maximize the signal-to-inference ratio, is shown to be preferable.
\end{abstract}

\section{INTRODUCTION}

The combination of multiple-antenna techniques, often collectively referred to as multiple-input multiple-output (MIMO), with orthogonal frequency division multiplexing (OFDM) is promising for next-generation broadband high data rate wireless systems [1], [2]. Application of these kind of systems is foreseen in environments with rich scattering, i.e., where the wireless channel is highly dispersive. In systems with high bandwidths, these multipath channels cause frequency selectivity and inter-symbol interference (ISI).

The frequency selectivity is effectively dealt with by OFDM by dividing the system bandwidth up into parallel subcarriers, all experiencing a frequency flat subchannel. To overcome the ISI most OFDM based systems apply a cyclic prefix (CP) in front of every symbol. This $\mathrm{CP}$, often also referred to as guard interval (GI), introduces redundancy, which is removed in the receiver, before data detection. In this way the influence of the ISI caused by the multipath channel can be largely reduced.

The CP, however, also significantly decreases the effective data rate of the system. It is, therefore, important that the ratio between the length of the $\mathrm{CP}$ and the number of carriers is minimized. One solution is to keep the $\mathrm{CP}$ length low compared to the channel impulse response (CIR) length. Then, however, ISI will possibly become the performance limiting factor and the placement of the discrete Fourier transform (DFT) window within the stream of received OFDM symbols, here referred to as symbol timing, becomes important. Note that symbol timing in previous literature is often referred to as fine timing, in contrast to coarse timing which indicates the packet detection.

Several frame timing approaches for single-input singleoutput (SISO) OFDM have been proposed previously in literature. Generally, they are based on a maximizing of a timing measure which is found by either correlation between repeated dedicated training symbols, see e.g. [3] and [4], or correlation between the redundant parts in the data symbols, see e.g. [5]. The limited accuracy of these algorithm makes their applicability to systems with short CP lengths questionable. Therefore, this paper discusses the use of techniques that are based on knowledge of the CIR, examples of which for SISO OFDM can be found in, e.g., [6]-[8].

Few publications treat the problem of symbol timing in multiple antenna OFDM systems, which indeed is different from the SISO problem since the received signal is now a sum of different signals propagating through different channels. One solution for MIMO OFDM symbol timing can be found in [9], where the peak in the cross correlation of the received and transmitted preamble symbols is used as timing reference. Is this paper we also use the estimate of the MIMO CIR to determine the optimal placement of the DFT window. Instead of searching the dominant path, as done in [9], we propose a method that attempts to optimize the signal-to-ISI ratio (SIR) of the received symbols used for data detection.

The layout of the paper is as follows. Section II describes the considered multiple antenna OFDM system. Subsequently, the estimation of the MIMO CIR is treated in Section III. Section IV extends the conventional approach to symbol timing, using the location of the strongest path in the CIR, for application in a MIMO system. Section V, subsequently, proposes a novel timing approach based on maximization of the SIR. In Section VI a sub-optimal algorithm with reduced computational complexity is proposed. The performance of the algorithms is tested by Monte Carlo simulations, the results of which are presented in Section VII. Finally, conclusions are drawn in Section VIII.

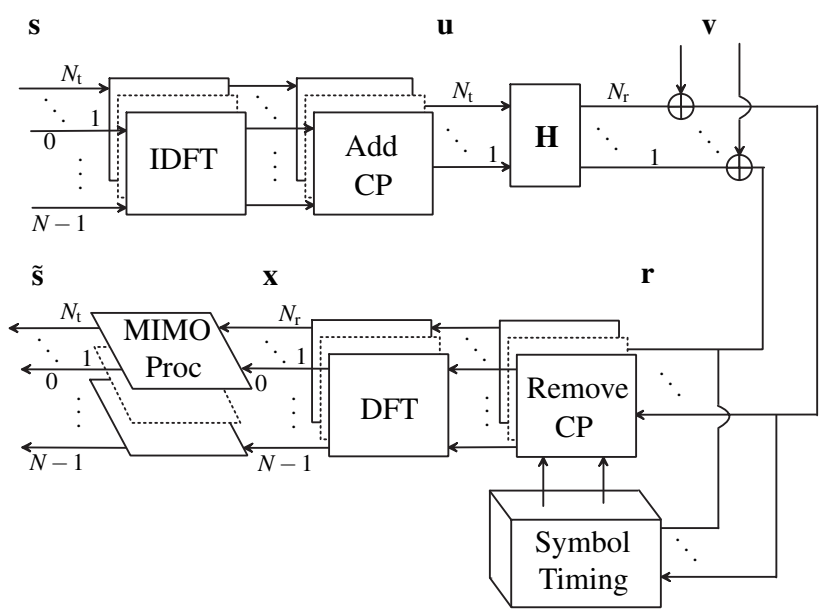

Fig. 1. Baseband model for a MIMO OFDM system applying symbol timing. 


\section{SYSTEM DESCRIPTION}

Consider a MIMO OFDM system with $N_{\mathrm{t}}$ transmitter (TX) and $N_{\mathrm{r}}$ receiver (RX) branches, $N$ subcarriers and a CP length of $N_{\mathrm{g}}$ samples, as schematically depicted in Fig. 1. The vector of (complex) data symbols in the frequency domain is denoted by $\mathrm{s}$ here, the size of which equals $N N_{\mathrm{t}} \times 1$. After the inverse discrete Fourier transform (IDFT) and the addition of the CP the $N_{\mathrm{s}} N_{\mathrm{t}} \times 1$ signal vector is given by $\mathbf{u}$, where the symbol length is denoted by $N_{\mathrm{s}}=N+N_{\mathrm{g}}$. The upconverted signal is transmitted through the wireless channel and downconverted at the RX, here modelled by transmission through the baseband equivalent channel $\mathbf{H}$. There the $N_{\mathrm{r}} \times 1$ received time-domain vector during sample time $n$ is expressed by

$$
\mathbf{r}[n]=\left[r_{1}[n], \ldots, r_{N_{\mathrm{r}}}[n]\right]^{T}=\sum_{l=0}^{L-1} \mathbf{H}[l] \mathbf{u}[n-l],
$$

where $\mathbf{u}[n]$ denotes the $N_{\mathrm{t}} \times 1$ transmitted vector during sample time $n, L$ is the length of the channel and ${ }^{T}$ denotes the matrix transpose. For the following it is useful to group these vectors per OFDM symbol. Therefore, we introduce the following short notation for the $n$th sample of the $m$ th OFDM symbol: $\mathbf{r}_{m}[n]=\mathbf{r}\left[(m-1) N_{\mathrm{s}}+n\right]$ and $\mathbf{u}_{m}[n]=\mathbf{u}\left[(m-1) N_{\mathrm{s}}+n\right]$. Using this notation, the expression for the received signal in (1) can be rewritten for the $n$th sample of the $m$ th received symbol vector as

$$
\begin{aligned}
\mathbf{r}_{m}[n] & =\left[r_{m, 1}[n], \ldots, r_{m, N_{\mathrm{r}}}[n]\right]^{T} \\
= & \sum_{l=0}^{n-1} \mathbf{H}[l] \mathbf{u}_{m}[n-l]+\sum_{l=0}^{L-n-1} \mathbf{H}[l+n] \mathbf{u}_{m-1}\left[N_{\mathrm{s}}-l\right]
\end{aligned}
$$

for $n=1, \ldots, N_{\mathrm{s}}$ and where we assumed $L \leq N_{\mathrm{s}}$. We can conclude from (2) that the received signal contains samples from the regarded and the previous symbol, i.e., the first term is the useful signal term and the second term is the ISI. The symbol timing now determines which $N$ out of $N_{\mathrm{s}}$ samples of $\mathbf{r}_{m}$ are used to determine the frequency domain signal vector $\mathbf{x}_{m}$. This vector is then used by the MIMO processing to estimate the transmitted data.

If the symbol timing is chosen to be $p_{n_{\mathrm{r}}}$, the $N$-dimensional vector input to the DFT for the $n_{\mathrm{r}}$ th $\mathrm{RX}$ is given by

$$
\begin{aligned}
\mathbf{r}_{m, n_{\mathrm{r}}}\left(p_{n_{\mathrm{r}}}\right) & =\left[r_{m, n_{\mathrm{r}}}\left[p_{n_{\mathrm{r}}}\right], \ldots, r_{m, n_{\mathrm{r}}}\left[p_{n_{\mathrm{r}}}+N-1\right]\right]^{T} \\
& =\mathbf{r}_{m, n_{\mathrm{r}}}^{\text {sig }}\left(p_{n_{\mathrm{r}}}\right)+\mathbf{r}_{m, n_{\mathrm{r}}}^{\text {isi }}\left(p_{n_{\mathrm{r}}}\right),
\end{aligned}
$$

where the $\mathbf{r}_{m, n_{\mathrm{r}}}^{\mathrm{sig}}\left(p_{n_{\mathrm{r}}}\right)$ and $\mathbf{r}_{m, n_{\mathrm{r}}}^{\mathrm{isi}}\left(p_{n_{\mathrm{r}}}\right)$ are the desired and ISI vector, respectively, and their structure is equal to that of (3). Their $n$th elements are given by

$$
\begin{aligned}
& r_{m, n_{\mathrm{r}}}^{\mathrm{sig}}\left[n, p_{n_{\mathrm{r}}}\right]=\sum_{l=0}^{p_{n_{\mathrm{r}}}+n-1} \mathbf{h}_{n_{\mathrm{r}}}[l] \mathbf{u}_{m}\left[p_{n_{\mathrm{r}}}+n-l\right], \\
& r_{m, n_{\mathrm{r}}}^{\mathrm{isi}}\left[n, p_{n_{\mathrm{r}}}\right]=\sum_{l=0}^{L-\left(p_{n_{\mathrm{r}}}+n\right)-1} \mathbf{h}_{n_{\mathrm{r}}}\left[l+p_{n_{\mathrm{r}}}+n\right] \mathbf{u}_{m-1}\left[N_{\mathrm{s}}-l\right]
\end{aligned}
$$

for $n=0, \ldots, N-1$, respectively. Here the $n_{\mathrm{r}}$ th row of $\mathbf{H}$ is denoted by $\mathbf{h}_{n_{\mathrm{r}}}$.
As a performance measure of the timing we now consider the power of the desired signal and ISI terms. Hereto we calculate the expected power value of the desired signal term for timing point $p$ and a given channel realisation $\mathbf{H}$. Since the DFT is applied per RX branch, it is derived here for the $n_{\mathrm{r}}$ th $\mathrm{RX}$ branch. For a timing point $p$, the expected signal power is given by

$$
\begin{aligned}
& P_{n_{\mathrm{r}}}^{\mathrm{sig}}(p)=\mathbb{E}\left[\left\{\mathbf{r}_{m, n_{\mathrm{r}}}^{\mathrm{sig}}(p)\right\}^{H} \mathbf{r}_{m, n_{\mathrm{r}}}^{\mathrm{sig}}(p) \mid \mathbf{h}_{n_{\mathrm{r}}}\right] \\
= & N \sigma_{\mathrm{u}}^{2} \sum_{k=0}^{p} \mathbf{h}_{n_{\mathrm{r}}}[k] \mathbf{h}_{n_{\mathrm{r}}}^{H}[k]+\sigma_{\mathrm{u}}^{2} \sum_{k=1}^{N+1}(N-k) \mathbf{h}_{n_{\mathrm{r}}}[k+p] \mathbf{h}_{n_{\mathrm{r}}}^{H}[k+p],
\end{aligned}
$$

where ${ }^{H}$ denotes the conjugate transpose and where the elements of $\mathbf{u}$ are assumed to be independently and identically distributed and to have zero-mean and a variance of $\sigma_{\mathrm{u}}^{2}$.

In a similar way the expected value of the ISI power can be derived as

$$
\begin{aligned}
& P_{n_{\mathrm{r}}}^{\mathrm{isi}}(p)=\mathbb{E}\left[\left\{\mathbf{r}_{m, n_{\mathrm{r}}}^{\mathrm{isi}}(p)\right\}^{H} \mathbf{r}_{m, n_{\mathrm{r}}}^{\mathrm{isi}}(p) \mid \mathbf{h}_{n_{\mathrm{r}}}\right] \\
= & N \sigma_{\mathrm{u}}^{2} \sum_{k=N+p}^{L-1} \mathbf{h}_{n_{\mathrm{r}}}[k] \mathbf{h}_{n_{\mathrm{r}}}^{H}[k]+\sigma_{\mathrm{u}}^{2} \sum_{k=1}^{N-1} k \mathbf{h}_{n_{\mathrm{r}}}[k+p] \mathbf{h}_{n_{\mathrm{r}}}^{H}[k+p] .
\end{aligned}
$$

The expressions for the wanted signal and ISI power, in (7) and (8), are defined for $1 \leq p \leq N_{\mathrm{g}}$. Note that for $p>N_{\mathrm{g}}$ these expressions change slightly, since samples of the next symbol will be included in the DFT window, also causing ISI.

Clearly the expressions for the expected power of the desired signal and ISI in (7) and (8) depend on the channel realization. Knowledge of the MIMO CIR is, therefore, required to be able to apply these expressions for symbol timing. In a practical system, however, full knowledge of the channel is often not available and an estimate of the channel has to be acquired, a subject that will be treated in the next section.

\section{CIR ESTIMATION}

Let us now consider a packet transmission, where the channel can be assumed to be quasi static, i.e., the channel is constant during the reception of a packet. For such a system often a piece of known data, i.e., preamble, is transmitted in front of the data part to enable estimation of the MIMO CIR, which is also required for MIMO detection. MIMO channel estimation and the design of an efficient preamble has been the subject of many contributions over the last few years, see e.g. [10]-[13], and details are, therefore, omitted here. Basically, the preamble has to provide orthogonality between the spatial streams with sequences that are shift-orthogonal to allow for estimation of the multipath channel. For such a preamble, a correlation with the transmitted preamble sequences at the RX will result in an estimate of the MIMO CIR.

Although the different channel estimation/preamble combinations will result in a different performance, we can generally write the resulting estimate of the $l$ th tap of the $N_{\mathrm{t}} \times N_{\mathrm{r}}$ MIMO CIR matrix $\mathbf{H}[l]$ by $\tilde{\mathbf{H}}[l]=\mathbf{H}[l]+\varepsilon[l]$, where $\varepsilon[l]$ is the estimation error in the $l$ th tap of the CIR, which is here assumed to 
be zero-mean circular symmetric complex Gaussian distributed with a variance of $\sigma_{\varepsilon}^{2}$. Clearly this variance decreases with increasing signal-to-noise ratio (SNR).

Sections IV to VI will exploit the knowledge of the MIMO CIR to determine the symbol timing. Although only an estimate is available, we will assume perfect channel knowledge in these sections. The impact of this assumption is tested by a numerical study in Section VII.

\section{Dominant Path Detection}

The straightforward method to determine the symbol timing is to relate the timing to the maximum path in the CIR. This is based on the observation that paths in the CIR with the smallest delay will generally experience the lowest attenuation. Generally an offset of several samples is applied to take into account possible smaller taps preceding the path with the maximum power in the CIR.

\section{A. $R X$-branch timing}

When this technique is applied for a MIMO system to calculate the symbol timing for one of the RX branches, i.e., $n_{\mathrm{r}}$, the estimated symbol timing point is given by

$$
p_{n_{\mathrm{r}}}=\arg \max _{k}\left\{\mathbf{h}_{n_{\mathrm{r}}}[k] \mathbf{h}_{n_{\mathrm{r}}}^{H}[k]\right\}-c+N_{\mathrm{g}} .
$$

where $c$ is the above mentioned offset parameter. Furthermore, the constant $N_{\mathrm{g}}$ is added since the outcome of the $\arg \max$ indicates the beginning of the OFDM symbol, rather than the beginning of the DFT window. This is also the case for (10), (13) and (14). It is noted that for the $R X$-branch timing every $\mathrm{RX}$ branch uses a separate symbol timing.

\section{B. Joint timing}

To make the processing in the MIMO RX less complex, a common symbol timing for the whole MIMO RX can be calculated. The timing point is then found as

$$
p=\arg \max _{k}\left\{\sum_{n_{\mathrm{r}}=1}^{N_{\mathrm{r}}} \mathbf{h}_{n_{\mathrm{r}}}[k] \mathbf{h}_{n_{\mathrm{r}}}^{H}[k]\right\}-c+N_{\mathrm{g}} .
$$

A similar approach for the joint symbol timing was previously proposed by the authors of [9].

\section{SIR OPTIMIZATION}

A more optimal approach to symbol timing in the case of perfect channel knowledge is proposed here. It maximizes the SIR at the input of the DFT, i.e., the method finds a timing point that minimizes the amount of ISI and maximizes the amount of signal falling into the DFT window.

\section{A. $R X$-branch timing}

For this approach the symbol timing point for the $n_{\mathrm{r}}$ th $\mathrm{RX}$ branch is found by

$$
p_{n_{\mathrm{r}}}=\arg \max _{p}\left\{P_{n_{\mathrm{r}}}^{\mathrm{sig}}(p) / P_{n_{\mathrm{r}}}^{\mathrm{isi}}(p)\right\} .
$$

where the expected signal and ISI power are given by (7) and (8), respectively. An alternative approach would be to maximize the desired signal power in (7) or to minimize the
ISI power in (8). Since this is very similar to (11), it is not treated in this paper.

\section{B. Joint timing}

For the case of a joint symbol timing for the entire MIMO receiver, the ratio of the total signal power and total ISI power is calculated. The symbol timing point is then found by

$$
p=\arg \max _{p}\left\{\sum_{n_{\mathrm{r}}=1}^{N_{\mathrm{r}}} P_{n_{\mathrm{r}}}^{\mathrm{sig}}(p) / \sum_{n_{\mathrm{r}}=1}^{N_{\mathrm{r}}} P_{n_{\mathrm{r}}}^{\mathrm{isi}}(p)\right\} .
$$

\section{REDUCED COMPLEXITY ALGORITHM}

A less computational complex algorithm, i.e., requiring less operations for implementation, was previously proposed for SISO OFDM in [6, pp.88-92] and [8], and finds the maximum of the convolution of the CIR powers with a rectangular window of length $N_{\mathrm{g}}$, i.e., the length of the guard interval. In doing so, the algorithm attempts to maximize the amount of ISI within the CP, i.e., minimizing the ISI within the DFT window.

\section{A. $R X$-branch timing}

In the extension for a MIMO system, the resulting timing point for the $n_{\mathrm{r}}$ th $\mathrm{RX}$ branch is found by

$$
p_{n_{\mathrm{r}}}=\arg \max _{p}\left\{\sum_{k=p}^{p+N_{\mathrm{g}}} \mathbf{h}_{n_{\mathrm{r}}}[k] \mathbf{h}_{n_{\mathrm{r}}}^{H}[k]\right\}+N_{\mathrm{g}} .
$$

\section{B. Joint timing}

The extension for joint timing is given by

$$
p=\arg \max _{p}\left\{\sum_{n_{\mathrm{r}}=1}^{N_{\mathrm{r}}} \sum_{k=p}^{p+N_{\mathrm{g}}} \mathbf{h}_{n_{\mathrm{r}}}[k] \mathbf{h}_{n_{\mathrm{r}}}^{H}[k]\right\}+N_{\mathrm{g}} .
$$

It is noted that this joint timing is similar to what was applied in the MIMO OFDM implementation discussed in [2].

\section{NUMERICAL RESULtS}

The performance of the algorithms discussed in Sections IV to VI is evaluated here. Hereto Monte Carlo simulations were performed for a $4 \times 4$ MIMO extension of the IEEE 802.11a standard [14], i.e., for $N=64$ and $N_{\mathrm{g}}=16$.

\section{A. Channel Modeling}

In the simulations two types of channels were applied: a single-cluster and a double-cluster channel, as schematically depicted in Fig. 2. The former relates to an indoor environment and the latter maps onto an outdoor scenario, e.g., a singlefrequency broadcasting system.

For both channels the clusters are assumed to have exponential decaying power-delay-profiles (PDPs) and the amplitude fading is assumed to be Rayleigh distributed. The normalized rms delay spread is defined per cluster and denoted by $\tau$. For the double-cluster model the distance between the start of the clusters is given by $\delta$. Furthermore, spatially uncorrelated MIMO channels were assumed. 


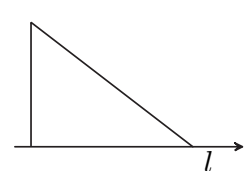

(a) single-cluster

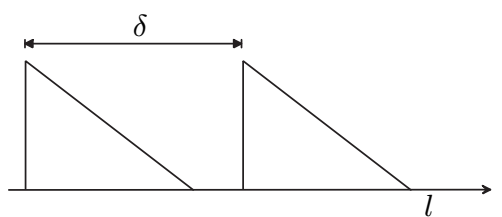

(b) double-cluster
Fig. 2. Schematic representation of the PDPs of the applied channel models.

\section{B. Performance evaluation}

In the following we will derive the performance of the symbol timing as function of the accuracy of the channel estimation. Therefore, we define the CIR-to-CIR-error ratio (CCER) for the $n_{\mathrm{r}}$ th $\mathrm{RX}$ branch as

$$
\operatorname{CCER}_{n_{\mathrm{r}}}=\frac{\mathbb{E}\left[\sum_{l=0}^{L-1} \boldsymbol{h}_{n_{\mathrm{r}}}[l] \boldsymbol{h}_{n_{\mathrm{r}}}^{H}[l]\right]}{\mathbb{E}\left[\sum_{l=0}^{L-1} \varepsilon_{n_{\mathrm{r}}}[l] \varepsilon_{n_{\mathrm{r}}}^{H}[l]\right]},
$$

which in fact equals the inverse of the normalized meansquared-error (MSE) of the MIMO CIR estimate for the $n_{\mathrm{r}}$ th branch. The CCER averaged over the different RX antennas is then denoted by CCER.

As a first measure of performance the MSE in estimation of the symbol timing for the single-cluster channel with $\tau=$ $N_{\mathrm{g}} / 2$ is regarded. The symbol timing algorithms are regarded for one of the RX branches. The timing error is defined as the difference between the estimated timing point and the timing that would result from (11) with ideal CIR knowledge, i.e., the timing that optimizes the SIR. The results as function of the CCER are depicted in Fig. 3. Since high rms delay spreads are regarded in these simulations, the offset parameter for the dominant path algorithm was always chosen to be $c=1$.

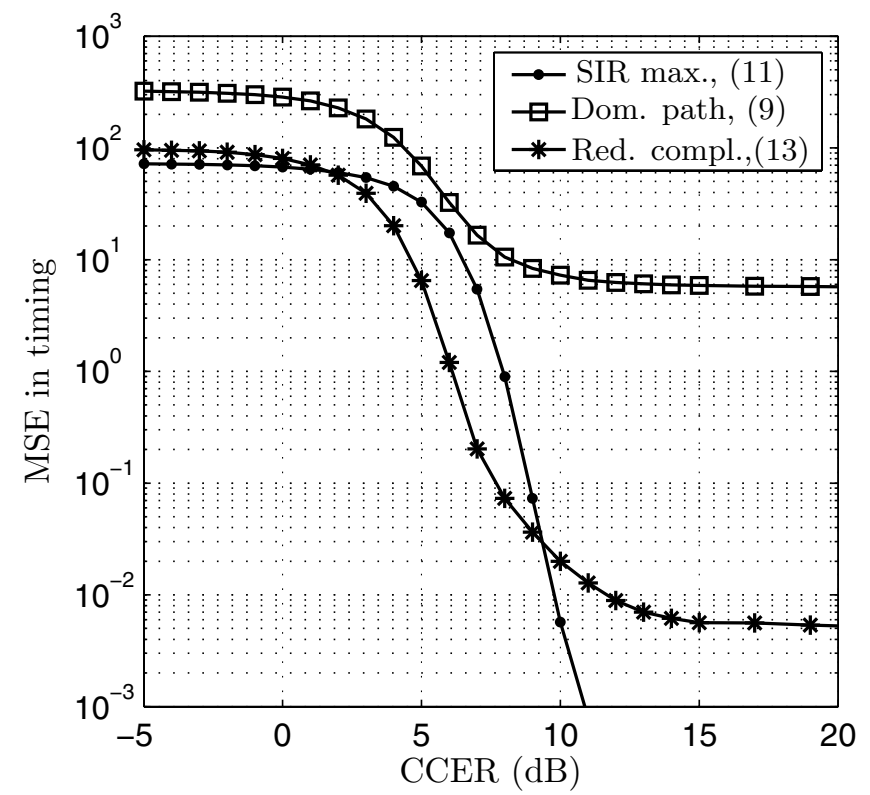

Fig. 3. MSE in symbol timing as function of the CCER for the different timing algorithms. Single-cluster channel, normalized rms delay spread $\tau=$ $N_{\mathrm{g}} / 2$.
It can be concluded from Fig. 3 that, in terms of MSE, the dominant path search algorithm of (9) performs worst over the whole CCER range. For low CCER values the other 2 algorithms show a comparable performance. At high CCER values the SIR maximization algorithm of (11) performs best. Although the reduced complexity algorithm of (13) performs best in the medium CCER range, it shows flooring for the high CCER range.

It is noted, however, that although the MSE in symbol timing gives a measure for the error in the timing, it does not show the degradation in performance due to this error. Therefore, the following figures will report the achieved SIR with a certain timing algorithm as function of the CCER. When the SIR has the same order of magnitude as or is larger than the experienced SNR, the system performance will be limited by the ISI.
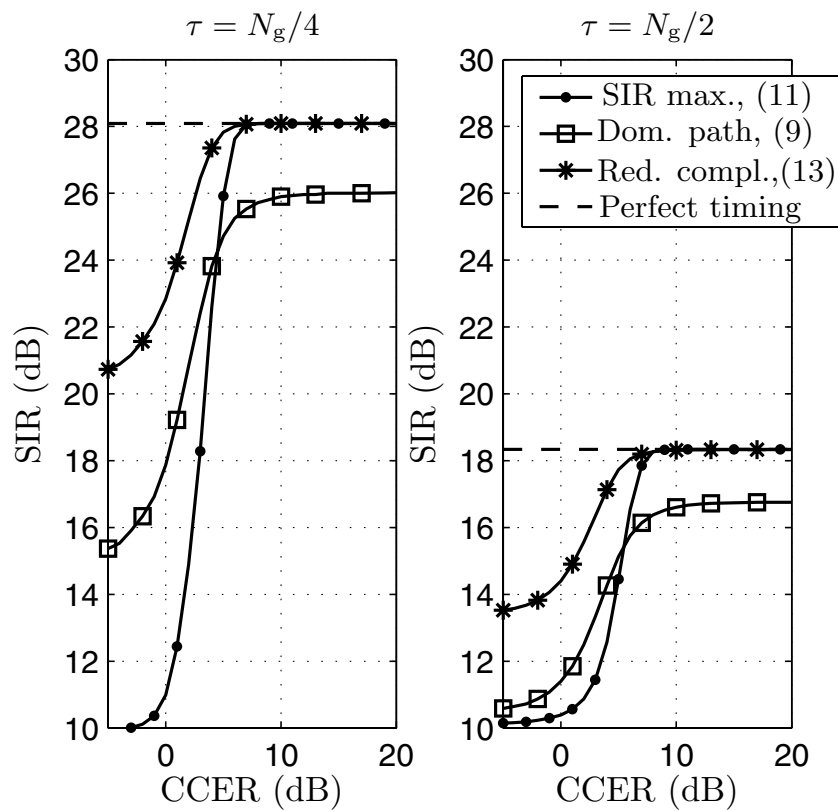

Fig. 4. SIR as function of the CCER for the different timing algorithms. Single-cluster channel.

In Fig. 4 the SIR performance is given for the single-cluster model for $\tau=N_{\mathrm{g}} / 4$ and $\tau=N_{\mathrm{g}} / 2$. The optimal SIR values obtained with perfect timing are depicted as dashed lines, at $28 \mathrm{~dB}$ and $18.3 \mathrm{~dB}$, respectively. The maximum achievable performance is smaller for $\tau=N_{\mathrm{g}} / 2$, since more ISI occurs due to the longer channel. It can be observed that the reduced complexity model achieves the bound for the lowest CCER value, closely followed by the SIR maximization method. It is observed that the dominant path search method shows flooring below the optimum for both cases, while it performs better than the SIR maximization algorithm for very inaccurate channel estimates.

To compare the performance of the joint and RX timing, the achievable SIRs are in Fig. 5 compared for a single-cluster channel with $\tau=3 N_{\mathrm{g}} / 8$. It can be concluded that for the SIR maximization the joint timing performs a little worse than the separate timing for low CCER values. For the reduced 


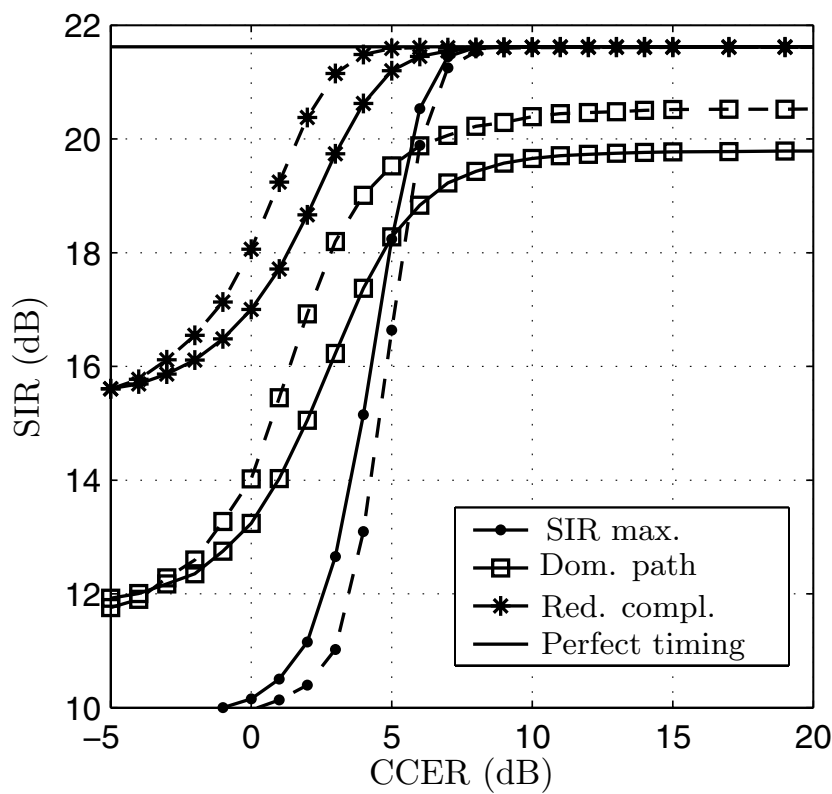

Fig. 5. SIR as function of the CCER for the different timing algorithms Single-cluster channel, $\tau=3 N_{\mathrm{g}} / 8$. RX-branch timing (solid lines) and joint timing (dashed lines).

complexity and dominant path detector this is the other way around. This is explained by the fact that these techniques work best for an exponentially decaying channel, which is better achieved for the joint timing, since the fading and noise are averaged out here.

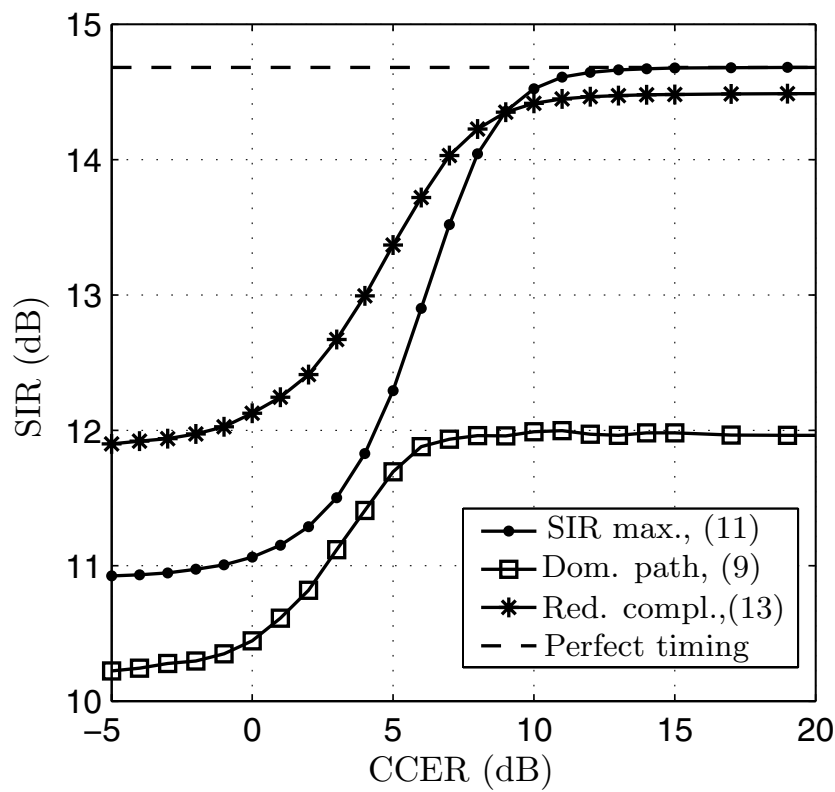

Fig. 6. SIR as function of the CCER for the different timing algorithms. Double-cluster channel, $\tau=N_{\mathrm{g}} / 4, \delta=9 N_{\mathrm{g}} / 10$.

Finally, Fig. 6 presents SIR results for the double-cluster model, where again per RX timing is considered. It is observed that the optimal performance is only achieved by the SIR maximization method. The dominant path search method shows severe flooring. From this result, and more results for the double-cluster model not shown here, it can be concluded that only the algorithm in (11) achieves the optimal performance for these kind of channels with two clusters and high clusters separation $\delta$. This can be explained by the fact that both other methods inherently assume that the power of the channel taps decreases as function of delay, which is not the case for the regarded double-cluster channel.

\section{CONCLUSions}

The problem of symbol timing in multiple antenna OFDM systems was considered in this paper. Extensions of OFDM symbol timing methods were proposed for application in MIMO systems. Additionally, a novel SIR maximization algorithm was presented. The performance of the different algorithms was tested by means of simulations with a singleand double-cluster channel model. It can be concluded that the reduced complexity algorithm performs superior, especially for low CIR accuracy. The SIR maximization method is, however, preferred when the channel is accurately known and when double-cluster channels occur.

\section{ACKNOWLEDGEMENTS}

This work was carried out within the B4 Broadband Radio@hand project, which is supported in part by the Dutch Ministry of Economic Affairs under grant BTS01063.

\section{REFERENCES}

[1] H. Sampath, S. Talwar, J. Tellado, V. Erceg, and A. Paulraj, "A fourth-generation MIMO-OFDM broadband wireless system: design, performance, and field trial results," IEEE Commun. Magazine, vol. 40, no. 9, pp. 143-149, Sep. 2002.

[2] A. van Zelst and T.C.W. Schenk, "Implementation of a MIMO OFDMbased wireless LAN system," IEEE Trans. on Sign. Proc., vol. 52, no. 2, pp. 483-494, Feb. 2004.

[3] T.M. Schmidl and D.C. Cox, "Robust frequency and timing synchronization for OFDM," IEEE Trans. on Communications, vol. 45, no. 12 , pp. 1613-1621, Dec. 1997

[4] T. Keller and L. Hanzo, "Orthogonal frequency division multiplex synchronisation techniques for wireless local area networks," in Proc. PIMRC'96, Taipei, Taiwan, 1996, pp. 963-967.

[5] J.J. van de Beek, M. Sandell, and P.O. Börjesson, "ML estimation of time and frequency offset in OFDM systems," IEEE Trans. on Signal Processing, vol. 45, no. 7, pp. 1800-1805, July 1997.

[6] R.D.J. van Nee and R. Prasad, OFDM for Wireless Multimedia Communications, Artech House Publishers, 2000.

[7] B.G. Yang, K.B. Letaief, R.S. Cheng, and Z. Cao, "Timing recovery for OFDM transmission," IEEE Journal on Selected Areas in Communications, vol. 18, no. 11, pp. 2278-2291, Nov. 2000.

[8] M. Speth, S.A. Fechtel, G. Fock, and H. Meyr, "Optimum receiver design for OFDM-based broadband transmission - part II: A case study," IEEE Trans. on Commun., vol. 49, no. 4, pp. 571-578, April 2001.

[9] A.N. Mody and G.L. Stüber, "Synchronization for MIMO OFDM systems," in Proc. IEEE Global Communications Conference 2001, Nov. 2001, vol. 1, pp. 509-513.

[10] E.G. Larsson and J. Li, "Preamble design for multiple-antenna OFDMbased WLANs with null subcarriers," IEEE Signal Processing Letters, vol. 8, no. 11, pp. 285-288, Nov. 2001.

[11] Y. Li, "Simplified channel estimation for OFDM systems with multiple transmit antennas," IEEE Trans. on Wireless Commun., vol. 1, no. 1, pp. 67-75, Jan. 2002.

[12] I. Barhumi, G. Leus, and M. Moonen, "Optimal training design for MIMO OFDM systems in mobile wireless channels," IEEE Trans. on Sign. Proc., vol. 51, no. 6, pp. 1615-1624, June 2003.

[13] T.C.W. Schenk and B. Driesen, "Backwards compatibility for MIMO systems based on IEEE 802.11a," in Proc. 10th International OFDMWorkshop 2005, Hamburg, Germany, Aug. 2005, pp. 109-113.

[14] IEEE 802.11 a standard, ISO/IEC 802-11:1999/Amd 1:2000(E). 